\title{
AN IMPROVED TALBOT METHOD FOR NUMERICAL LAPLACE TRANSFORM INVERSION
}

\author{
BENEDICT DINGFELDER AND J.A.C. WEIDEMAN
}

\begin{abstract}
Aвstract. The classical Talbot method for the computation of the inverse Laplace transform is improved for the case where the transform is analytic in the complex plane except for the negative real axis. First, by using a truncated Talbot contour rather than the classical contour that goes to infinity in the left half-plane, faster convergence is achieved. Second, a control mechanism for improving numerical stability is introduced.
\end{abstract}

\section{INTRODUCTION}

Let $f(t)$ be real-valued and piecewise continuous on $[0, \infty)$ and of exponential order $f=\mathcal{O}\left(e^{\gamma_{0} t}\right), t \rightarrow \infty$, for some real constant $\gamma_{0}$. Then the Laplace transform of $f(t)$ is defined by

$$
F(z)=\int_{0}^{\infty} e^{-z t} f(t) d t, \quad \operatorname{Re} z>\gamma_{0} .
$$

This paper deals with the numerical solution of the inverse problem, i.e., given $F(z)$, compute $f(t)$ at a specified value of $t$. The function $F(z)$ is analytic in the half-plane $\operatorname{Re} z>\gamma_{0}$, and if it can be evaluated in the complex plane (as opposed to just on the real axis), this analyticity can and should be exploited by good numerical methods. One such method is numerical quadrature applied to the inverse formula

$$
f(t)=\frac{1}{2 \pi i} \int_{\gamma-i \infty}^{\gamma+i \infty} e^{z t} F(z) d z, \quad \operatorname{Re} \gamma>\gamma_{0},
$$

known as the Bromwich integral. Two effective quadrature rules are the simple trapezoidal and midpoint rules, used in combination with contour deformation as first suggested in [But57].

The purpose of the contour deformation is to exploit the exponential factor in (1). In particular, assume the path of integration in (1) can be deformed to a Hankel contour, i.e., a contour whose real part begins at negative infinity in the third quadrant, then winds around all singularities and terminates with the real part again going to negative infinity in the second quadrant. Examples are shown in Figure 1 below. On such contours the exponential factor causes a rapid decay, which makes the integral particularly suitable for approximation by the trapezoidal or midpoint rules. The contour deformation can be justified by Cauchy's theorem, provided the contour remains in the domain of analyticity of $F(z)$. Some mild restrictions on the decay of $F(z)$ in the left half-plane are also required; for sufficient conditions, see [Tal79, TWSo6]. 
Suppose such a Hankel contour can be parameterized by

$$
C: z=z(\theta), \quad-\pi \leqslant \theta \leqslant \pi,
$$

where $\operatorname{Re} z( \pm \pi)=-\infty$. Then

$$
f(t)=\frac{1}{2 \pi i} \int_{C} e^{z t} F(z) d z=\frac{1}{2 \pi i} \int_{-\pi}^{\pi} e^{z(\theta) t} F(z(\theta)) z^{\prime}(\theta) d \theta .
$$

We approximate the latter integral by the $N$-panel midpoint rule with uniform spacing $h=2 \pi / N$, which yields

$$
f(t) \approx \frac{1}{N i} \sum_{k=1}^{N} e^{z\left(\theta_{k}\right) t} F\left(z\left(\theta_{k}\right)\right) z^{\prime}\left(\theta_{k}\right), \quad \theta_{k}=-\pi+\left(k-\frac{1}{2}\right) h .
$$

If the contour (2) is symmetric with respect to the real axis, and if $F(\bar{z})=\overline{F(z)}$ (which is the case for $f(t)$ real-valued), then half of the transform evaluations can be saved. That is, one needs to consider only quadrature nodes in the upper (or lower) half-plane. When comparing numerical results it is important to keep this point in mind as several other papers, including [Duf93, Weio6, WTo7], consider quadrature rules with a total of $2 N$ nodes.

Popular contours $C$ are the parabola [But57, GMo5] and the hyperbola [GMo5, LFPo4, SSTo3], as well as the cotangent contour introduced by Talbot in [Tal79]. Here we consider the Talbot contour in the form

$$
z(\theta)=\frac{N}{t} \zeta(\theta), \quad \zeta(\theta)=-\sigma+\mu \theta \cot (\alpha \theta)+v i \theta, \quad-\pi \leqslant \theta \leqslant \pi,
$$

where $\sigma, \mu, v$ and $\alpha$ are constants to be specified by the user. In the original Talbot contour the parameter $\alpha$ did not appear, i.e., $\alpha=1$. For reasons outlined below, the modification we propose is to consider $0<\alpha<1$, in which case we refer to (5) as the modified Talbot contour. The scaling factor $N / t$ was introduced in the original paper [Tal79] and also used in [AV04, Weio6]. Assuming a fixed value of $t$, this is the appropriate scaling for maximizing the convergence rate in the trapezoidal/midpoint approximation as $N \rightarrow \infty$.

The practical issue addressed in this paper is the choice of the parameters $\sigma, \mu, v$ and $\alpha$ in (5). In his original paper [Tal79], Talbot suggested parameter choices in the case $\alpha=1$, for various configurations of the singularities of the transform $F(z)$. On the other hand, in [AVo4] a simpler approach was proposed that fixes $\sigma=0$, $\alpha=1$, and $\mu=v=0.2$ in (5) regardless of the singularity locations. (Note that the parameter 0.2 is the constant $\frac{2}{5}$ in the contour of [AVo4], when making allowance for the $N$ vs. $2 N$ convention as mentioned below (4)).

Existing software codes include Algorithm 682, which is a Fortran package that implements Talbot's original contour and parameter selection strategy [MR9o]. Alternatively, the parameter choices of $\left[\mathrm{AVo}_{0}\right]$ were incorporated in a Mathematica code [AVo2] as well as a MATLAB code [McC13].

These software implementations work well for many transforms. For the special case when all the singularities of $F(z)$ are located on the negative real axis, however, one can choose the parameters better, as argued in [Weio6]. By using a saddle point analysis, optimal parameters for the contour (5), with $\alpha=1$, were derived that predict a convergence rate of $\mathcal{O}\left(e^{-0.949 N}\right)$ as $N \rightarrow \infty$ for fixed $t>0$. An application of the same saddle point analysis to the contour of [AVo4] shows 
that the convergence rate in this case is slightly weaker, namely $\mathcal{O}\left(e^{-0.676 N}\right)$. This correlates well with the figure $\mathcal{O}\left(10^{-0.6 M}\right)$ obtained by numerical experimentation in [AVo4], when $M=N / 2$.

Somewhat surprisingly, simpler contours such as the parabola and hyperbola actually yield faster convergence than Talbot's original contour, namely $\mathcal{O}\left(e^{-1.047 N}\right)$ and $\mathcal{O}\left(e^{-1.176 N}\right)$ respectively; see [WTo7]. However, if one introduces the parameter $\alpha$ in (5) the convergence rate of the Talbot method can be improved to $\mathcal{O}\left(e^{-1.358 N}\right)$, which is significantly better than any of the other convergence rates cited. The above convergence rates, converted to base-10, are summarized in Table 1.

TABLE 1. Convergence rates cited in the literature. The estimates apply to transforms whose singularities are restricted to the negative real axis, such as $F(z)=(z+1)^{-1}$ or $z^{-1 / 2}$. Here $M=N / 2$, where $N$ is the total number of nodes in the quadrature sum (4); in other words, $M$ is the number of transform evaluations. The hyperbola and the modified Talbot contour are the only contours among these that gain at least one decimal digit per transform evaluation.

\begin{tabular}{ccc} 
Contour & Convergence & Reference \\
\hline Talbot & $10^{-0.6 M}$ & {$[$ AVo4] } \\
Talbot & $10^{-0.8 M}$ & {$[$ Weio6] } \\
Parabola & $10^{-0.9 M}$ & [WTo7] \\
Hyperbola & $10^{-1.0 M}$ & [WTo7] \\
Modified Talbot & $10^{-1.2 M}$ & [this paper]
\end{tabular}

Optimal parameters that achieve this $\mathcal{O}\left(e^{-1.358 N}\right)$ convergence rate were derived by one of the authors of the present paper, and reported in [TWSo6], later to be included in text books such as [Stro7, Sect. 5.3]. Details of the derivation have not been published, however. Having simplified and improved the derivation recently, we now present the details here.

The main considerations are summarized in Figure 1. The best contour has to strike a balance between passing too close to the singularities, or too far from them in which case the exponential factor in (3) becomes too large. The quadrature nodes on the best contour should also extend just far enough into the left half-plane to reach the desired accuracy but their contributions should not be negligibly small.

This discussion gives a clue as to why it is advantageous to introduce the parameter $\alpha$ in (5). Because $\operatorname{Re} z( \pm \pi)=-\infty$ in the original Talbot method, too many nodes end up far out in the left half-plane where they make no contribution to the quadrature sum (4). We acknowledge [Treo6] for this observation, and the suggestion to consider a truncated Talbot contour instead.

On a technical note, an error is committed when the truncated Talbot contour terminates at a finite location in the left half-plane. In the original derivation [Tal79], the path is closed at $\operatorname{Re}(z)=-\infty$. Because of the $e^{z t}$ factor, however, this error is of a similar magnitude as the truncation error-effectively all contributions to the left of the dash-dot curve in Figure 1 are ignored.

The above discussion also makes it clear that the optimal contour parameters are determined by the location of the singularities of $F(z)$. Clearly "one-size-fits-all" 


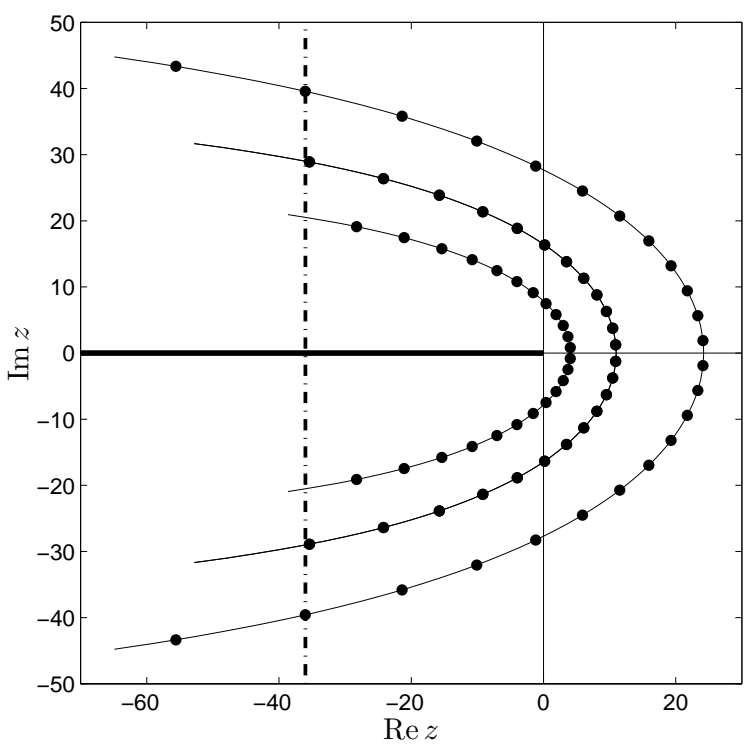

FigurE 1. Three possible Talbot contours (5), each shown with $N=24$ nodes in the midpoint rule. The middle contour is close to optimal, in the case where $t=1$ and the singularities of $F(z)$ are located on the negative real axis. Its outermost conjugate pair of nodes reaches right up to the dash-dot curve, where the magnitude of the exponential factor in (3) becomes less than the desired accuracy (here taken to be machine precision, $\epsilon \approx 2.2 \times 10^{-16}$ ). The inner contour is suboptimal because (a) its outermost two nodes do not reach quite up to the dash-dot curve and (b) it passes too close to the singularities. The outer contour is suboptimal because (a) the contributions of the outermost pair of nodes are less than the desired accuracy and therefore wasteful, and (b) the exponential factor in (3) is too large in the right half-plane.

parameter selections cannot be optimal in all situations. Below we continue to focus on the case when the singularities of $F(z)$ are located on the negative real axis. Although this restricts the class of transforms significantly, this case is sufficiently important in applications to justify such a study. For example, when solving certain parabolic PDEs, singularities on the negative real axis is an appropriate assumption to make; see Section 4 .

We conclude by commenting on the scaling factor $N / t$ in (5), which has a few negative consequences. First, because the nodes $z\left(\theta_{k}\right)$ depend on $N$, function values cannot be re-used as $N$ is increased. The increased convergence rate compensates for this, however. Second, for fixed $t$ the contour can move far into the right halfplane as $N$ is increased. Because of the exponential factor in (1), the terms in the summation can become large and hence suffer from floating-point cancelation errors. We address this issue in Section 3. Third, because the quadrature nodes depend on $t$ as well, the same contour cannot be used for two or more distinct values of $t$. In this case the method would be inefficient when the transform $F(z)$ is expensive to evaluate. We shall not address the latter issue here, but note that for 
the case $\alpha=1$ it was considered in [Riz95] and for the parabolic and hyperbolic contours it was considered in [WTo7].

The outline of the paper is as follows: In Section 2 we present the main error analysis that leads to the proposed values of $\sigma, \mu, v$ and $\alpha$. The analysis is similar to the one given in [Weio6]. It is slightly more intricate because of the additional parameter $\alpha$, but at the same time we have made some improvements that simplify the analysis. A strategy for improving numerical stability for large $N$ is discussed in Section 3. Like the analysis of Section 2, it is restricted to the case when the singularities of $F(z)$ are located on the negative real axis. Section 4 contains the numerical tests, where theory and experiment are compared.

\section{ERROR ESTIMATES}

Our basic error estimate is a well-known contour integral formula for the error in the trapezoidal rule approximation of functions that are analytic, periodic, and real-valued; see for example [Kreg8, Thm. 9.28]. We retain the property of analyticity but extend the theorem as follows: (A) we consider non-periodicity, (B) we consider complex-valued functions, and (C) we consider the midpoint rule rather than the trapezoidal rule. (A) is necessary because the integrand in (3) is not periodic. (B) is necessary because the integrand is complex-valued, and has very different analytic properties in the upper and lower half-planes. (C) is not really necessary, only a matter of convenience. All three of these modifications can be readily incorporated into the proof given in [Kre98, Thm. 9.28], which is an elementary application of the residue theorem. We omit the details.

Theorem 2.1. Let $\theta_{k}$ be defined as in (4). If $g: D \rightarrow \mathbb{C}$ is analytic in the rectangle $D=\{\theta \in \mathbb{C}:-\pi<\operatorname{Re} \theta<\pi$ and $-d<\operatorname{Im} \theta<c\}$ where $c, d>0$, then

$$
\int_{-\pi}^{\pi} g(\theta) d \theta-\frac{2 \pi}{N} \sum_{k=1}^{N} g\left(\theta_{k}\right)=E_{+}(\gamma)+E_{-}(\delta) .
$$

Here

$$
E_{+}(\gamma)=\frac{1}{2}\left(\int_{-\pi}^{-\pi+i \gamma}+\int_{-\pi+i \gamma}^{\pi+i \gamma}+\int_{\pi+i \gamma}^{\pi}\right)\left(1+i \tan \left(\frac{N \theta}{2}\right)\right) g(\theta) d \theta
$$

and

$$
E_{-}(\delta)=\frac{1}{2}\left(\int_{-\pi}^{-\pi-i \delta}+\int_{-\pi-i \delta}^{\pi-i \delta}+\int_{\pi-i \delta}^{\pi}\right)\left(1-i \tan \left(\frac{N \theta}{2}\right)\right) g(\theta) d \theta
$$

for all $0<\gamma<c, 0<\delta<d$ and $N$ even. For odd $N$, replace the $\tan (N \theta / 2)$ with $-\cot (N \theta / 2)$.

The contours of integration for computing the error integrals $E_{+}(\gamma)$ and $E_{-}(\delta)$ are shown in the left diagram of Figure 2. We remark that if $g(\theta)$ is real-valued on the real axis, then $g(\bar{\theta})=\overline{g(\theta)}$ and $d$ and $c$ can be taken to be equal. When it is moreover $2 \pi$-periodic, the contributions on the sides $\operatorname{Re} \theta= \pm \pi$ cancel. In that case, if we define $\mathrm{E}(\gamma)=\mathrm{E}_{+}(\gamma)+\mathrm{E}_{-}(\gamma)$, then

$$
\mathrm{E}(\gamma)=\operatorname{Re} \int_{-\pi+i \gamma}^{\pi+i \gamma}\left(1+i \tan \left(\frac{N \theta}{2}\right)\right) g(\theta) d \theta \text {. }
$$


Here, and below, we assume $N$ even but the analysis for odd $N$ is similar. By analyzing the behavior of the tangent function in the complex plane, this can be bounded by [Kre98, Thm. 9.28]

$$
|\mathrm{E}(\gamma)| \leqslant \frac{4 \pi \mathcal{M}}{e^{c N}-1}
$$

where $\mathcal{M}$ is a bound on the magnitude of $g$ in the domain $D$. For a given value of $N$, two quantities control the size of the error: $c$, which is the half-width of the rectangle of analyticity, and $\mathcal{M}$, which is governed by the growth of $g$ in the complex plane.

Here we apply the error formula of Theorem 2.1 to the case

$$
g(\theta)=\frac{1}{2 \pi i} e^{z(\theta) t} F(z(\theta)) z^{\prime}(\theta), \quad-\pi \leqslant \theta \leqslant \pi,
$$

where $F(z)$ is the transform to be inverted. This is not a real-valued function, which is why the rectangle of integration in Figure 2 is not shown symmetric with respect to the real axis. As we shall see (and similar observations were made in [Weio6, WTo7]), in the upper half-plane the top edge of the rectangle is restricted by the singularities of $F(z(\theta))$, while in the lower half-plane the restriction is the size of $e^{z(\theta) t}$.

To keep the analysis tractable, we limit our discussion primarily to the model transform

$$
F(z)=(z+\lambda)^{-1}, \quad \lambda>0,
$$

i.e., singularities on the negative real axis only. In Section 4 we shall consider a matrix version of this transform when considering applications to PDEs.
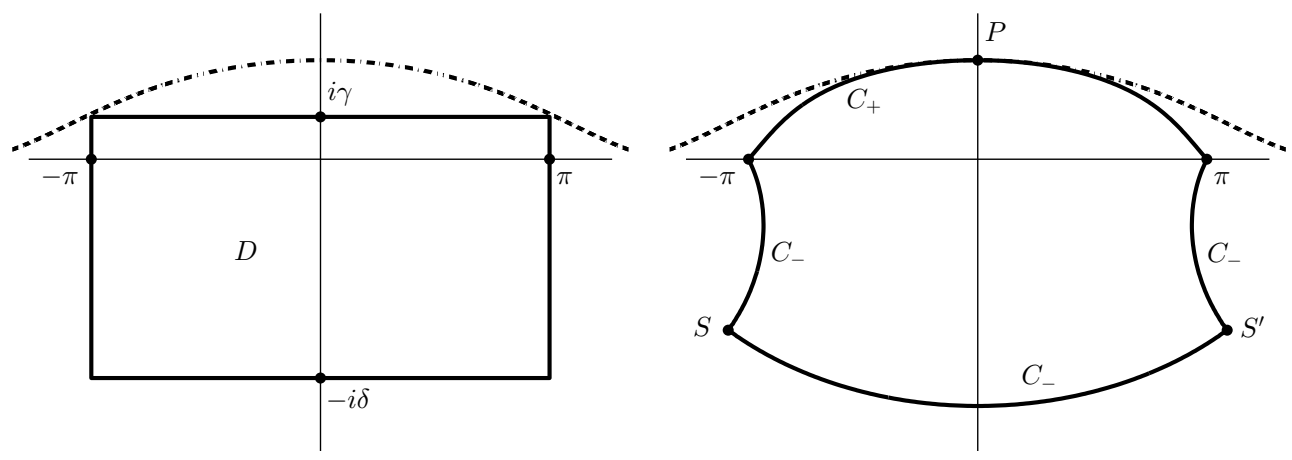

FIGURE 2. Left: Rectangular contour in the complex $\theta$-plane for evaluating the error integrals $\mathrm{E}_{+}(\gamma)$ and $\mathrm{E}_{-}(\delta)$ of Thm. 2.1. Right: Deformed contour, along which the magnitudes of the integrands of $E_{+}(\gamma)$ and $E_{-}(\delta)$ are approximately constant. The dash-dot curve in both diagrams shows a possible image of the negative real axis of the $z$-plane, where singularities might be located. The point $P$ is defined by the first two equations in (11) and the points $S$ and $S^{\prime}$ by the first two equations in (13).

For (7) and similar transforms, we can estimate the error using Theorem 2.1 and the rectangular contour shown on the left in Figure 2. For a better estimate, one can try and deform the contour as shown on the right in Figure 2. 
To explain how this can be done, consider the error integrals in Theorem 2.1. Ignoring the constant factors $1 / 2$ in those integrals, as well as the factor $1 /(2 \pi i)$ in (6), the error formulas can be expressed as

$$
E_{ \pm}=\int_{C_{ \pm}} e^{N h_{ \pm}(\theta)} d \theta
$$

where

$$
h_{ \pm}(\theta)=\zeta(\theta)+N^{-1}\left(\log \left(1 \pm i \tan \left(\frac{N \theta}{2}\right)\right)+\log F(z(\theta))+\log z^{\prime}(\theta)\right),
$$

and $C_{+}$and $C_{-}$refer to contours in the upper and lower half-planes, respectively. Our strategy is now as follows: suppose it is possible to deform the rectangle on the left in Figure 2 to a contour along which $\operatorname{Re} h_{ \pm}(\theta)=-c$ for some positive constant $c$. Then

$$
C_{ \pm}: \operatorname{Re} h_{ \pm}(\theta)=-c \quad \Longrightarrow \quad\left|E_{ \pm}\right|=O\left(e^{-c N}\right),
$$

and the value of $c$ can be maximized by picking the best among all such contours $C_{ \pm}$.

The function $h_{ \pm}(\theta)$ defined above is too complicated to analyze in this manner, and we make a few approximations in the case $N \gg 1$. First, we drop the terms involving $F(z(\theta))$ and $z^{\prime}(\theta)$. This means $F(z(\theta))$ should not be zero nor exponentially small on any part of the contours $C_{ \pm}$, and likewise $z^{\prime}(\theta) \neq 0$. Regarding the term involving the tangent function in $h_{ \pm}(\theta)$, we shall approximate it for $N \gg 1$ as follows. By converting the tangent function to exponentials one obtains, with $\theta=x+i y$,

$$
1 \pm i \tan \left(\frac{N}{2}(x+i y)\right)=\frac{2 e^{\mp \frac{1}{2} N y} e^{ \pm \frac{1}{2} i N x}}{e^{-\frac{1}{2} N y} e^{\frac{1}{2} i N x}+e^{\frac{1}{2} N y} e^{-\frac{1}{2} i N x}} .
$$

Taking the logarithm yields

$$
\log \left(1 \pm i \tan \left(\frac{N \theta}{2}\right)\right) \sim \mp N y, \quad N \rightarrow \infty,
$$

valid for fixed, nonzero $y$. The upper and lower sign choices correspond to $y>0$ and $y<0$, respectively. Accordingly, we approximate the contour $\operatorname{Re} h_{ \pm}(\theta)=-c$ in (8) by

$$
C_{ \pm}: \quad \operatorname{Re} \zeta(x+i y) \mp y=-c .
$$

First, consider the upper half of the $\theta$-plane. As mentioned above, the error contour $C_{+}$is restricted by the location of the singularities of $F(z(\theta))$ in the upper half of the $\theta$-plane. For now, consider the model problem (7). Its pole is located at $z=-\lambda$, or $\zeta=-\lambda t / N$. In keeping with the limit $N \rightarrow \infty, t$ fixed, we consider therefore $\zeta=0$. The same argument will apply if there is a branch-cut on the negative real $z$-axis, for in practice we need to consider only a finite section of the branch-cut (e.g., the section between the origin and the dashed line in Figure 1).

We therefore need to examine the zeros of $\zeta(\theta)$, i.e.,

$$
-\sigma+\mu \theta \cot (\alpha \theta)+v i \theta=0 .
$$

It is no easy task to establish theoretically how the complex roots of this equation depend on all possible parameter values. We therefore resorted to numerical rootfinding, restricting searches to the strip $-\pi \leqslant \operatorname{Re} \theta \leqslant \pi$, which is of interest here. This revealed the following: For certain parameter choices there are roots 
in the lower half of the $\theta$-plane. For other parameter choices the roots are in the upper half-plane, located symmetrically with respect to the imaginary $\theta$-axis. For yet other parameter values the roots are located precisely on the imaginary $\theta$-axis, with the one closest to the real axis the critical one that limits the contour $C_{+}$. Using such numerical experimentation and keeping in mind that $\zeta^{\prime}(\theta)$ may not be 0 as remarked below (8), we came to the same conclusion as [Weio6]. Namely, the configuration that gives the widest domain of analyticity in the upper half-plane occurs when the critical root (shown as the point marked $P$ in Figure 2) is located on the positive imaginary $\theta$-axis and is of double multiplicity.

This leads to the two equations $\zeta(i y)=0, \zeta^{\prime}(i y)=0$ for some $y>0$. By considering (10), we conclude that $y=c$. If we further let $\theta \rightarrow \pm \pi$ on the contour $C_{+}$, we get $\zeta( \pm \pi)=-c$, which is by symmetry just one equation, not two. In summary, in the upper half-plane we require that

$$
C_{+}: \quad \zeta(i c)=0, \quad \zeta^{\prime}(i c)=0, \quad \zeta(\pi)=-c .
$$

By using these three equations, it is possible to solve for $(\sigma, \mu, v)$ in terms of $(\alpha, c)$, namely

$$
\sigma=2 \alpha c^{2} B, \quad \mu=2 \sinh ^{2}(\alpha c) B, \quad v=(\sinh (2 \alpha c)-2 \alpha c) B,
$$

where

$$
B=\frac{c \sin ^{2}(\alpha \pi)}{2 \alpha c^{2} \sin ^{2}(\alpha \pi)-\pi \sin (2 \alpha \pi) \sinh ^{2}(\alpha c)} .
$$

It remains to fix the parameters $\alpha$ and $c$. For this, we consider the lower half of the $\theta$-plane. Here the contour $C_{-}$is not restricted by analyticity but by the size of the exponential factor in (3). Again numerical experimentation confirmed the findings of [Weio6], namely that there are two saddle points in the lower half of the $\theta$-plane that are critical. (These are the points marked $S$ and $S^{\prime}$ in Figure 2.) By making $C_{-}$pass through these saddle points, we can enclose a region as big as possible.
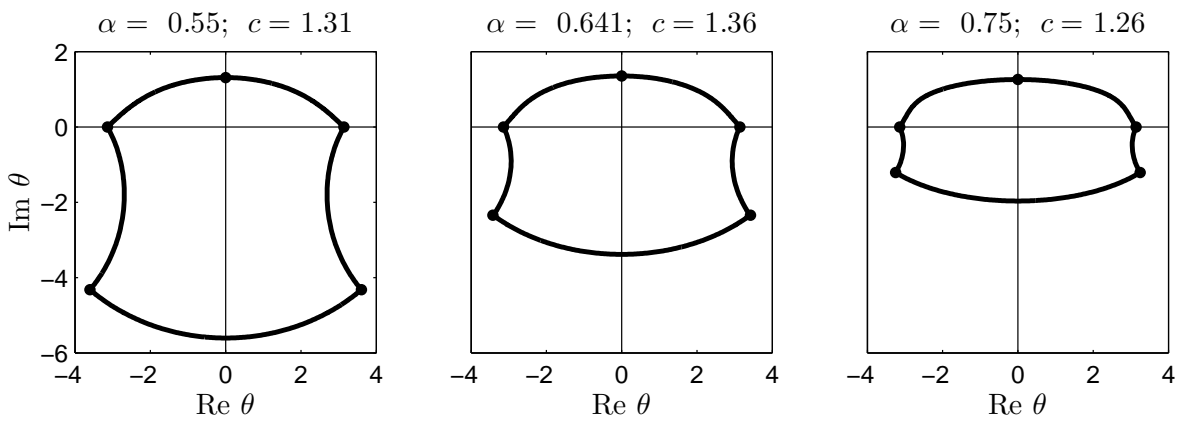

FIGURE 3. Three possible deformations of the rectangle of Figure 2. The middle one maximizes the value of $c$, which is both the value of the intersection on the positive imaginary axis and the decay rate in the error estimate $E=\mathcal{O}\left(e^{-c N}\right)$. 
Denote the saddle point locations by $\theta=x_{s}+i y_{s}$. By inserting this into (10), and taking partial derivatives, yields

$$
C_{-}: \quad \operatorname{Re} \zeta^{\prime}\left(x_{s}+i y_{s}\right)=0, \quad \operatorname{Im} \zeta^{\prime}\left(x_{s}+i y_{s}\right)=1, \quad \operatorname{Re} \zeta\left(x_{s}+i y_{s}\right)+y_{s}=-c . \quad \text { (13) }
$$

We insert the expressions for $\sigma, \mu$ and $v$ from (12) and fix a value of $\alpha$. Assuming a solution exists, the three equations (13) can then be solved numerically for the unknowns $x_{s}, y_{s}$, and $c$. By varying $\alpha$, the value of $c$ can thus be maximized.

We made no attempt to establish theoretical existence of a solution of the equations (13), but direct numerical computation indicates that such a solution exists for $\alpha$ in the interval $[0.51,0.82]$, roughly. Some of these configurations are shown in Figure 3, with the middle one corresponding to the maximum value of $c$. We computed this value of $c$ using a univariate optimization code, and found

$$
\alpha=0.6407 ; \quad c=1.3580 .
$$

Substitution of these two values into (12) yields the modified Talbot contour

$$
z(\theta)=\frac{N}{t}(-0.6122+0.5017 \theta \cot (0.6407 \theta)+0.2645 i \theta), \quad-\pi \leqslant \theta \leqslant \pi,
$$

as reported in [TWSo6]. For completeness, we record that the corresponding saddle points are located at $x_{s}+i y_{s}= \pm 3.4208-2.3438 i$, correct to all digits shown.

An alternative contour in which the cotangent is replaced by a rational function was respectively suggested and analyzed in [Tal79] and [Weio6]. Applying the same strategy as outlined above, we derived

$$
z(\theta)=\frac{N}{t}\left(0.1446+\frac{3.0232 \theta^{2}}{\theta^{2}-3.0767 \pi^{2}}+0.2339 i \theta\right), \quad-\pi \leqslant \theta \leqslant \pi .
$$

This rational contour is virtually as good as the cotangent contour, as it gives a convergence rate of $\mathcal{O}\left(e^{-1.311 N}\right)$, compared to the $\mathcal{O}\left(e^{-1.358 N}\right)$ of (15). With either contour, an accuracy of close to machine precision $\epsilon \approx 2.2 \times 10^{-16}$ can be reached for values of $N$ around 26 or 28 , i.e., about 13 or 14 transform evaluations.

We emphasize that we have assumed in this section that all singularities of $F(z)$ are located on the negative real axis, and $F(z)$ is not exponentially small as discussed below (8). We also ignored the effects of roundoff error, which we consider next.

\section{ROUNDOFF ERROR CONTROL}

In the analysis of the previous section we explicitly assumed that $N \gg 1$, but tacitly assumed infinite precision arithmetic. When $N$ gets large, however, the contours (15) and (16) move far into the right half-plane. The exponential factor in (4) gets big, and there is loss of floating-point precision in the summation. Fortunately, for the theory of the previous section to be applicable, $N$ needs to be only moderately large (we shall see that $N=20$ or even $N=10$ is adequate). There is therefore a range of values of $N$, say $N<N_{*}$, where the error estimates of the previous section are accurate. In the present section we estimate the critical value of $N=N_{*}$ where roundoff error becomes a factor, and we propose an alternate set of parameters to improve stability when $N>N_{*}$. 
Using the same arguments as presented in [Weiro] for the parabolic contour, we model the error caused by finite precision by

$$
R=\mathcal{O}\left(\epsilon e^{z(0) t}\right)=\mathcal{O}\left(\epsilon e^{N \zeta(0)}\right) .
$$

Here $\epsilon$ is the unit roundoff.

Let the implied constants in (8) and (17) be denoted by $k_{1}$ and $k_{2}$, respectively. Then the roundoff error becomes significant when

$$
k_{1} e^{-c N}=k_{2} \epsilon e^{N \zeta(0)} \Longrightarrow c+\zeta(0)+N^{-1} \log \left(\epsilon / k_{0}\right)=0,
$$

where we defined $k_{0}=k_{1} / k_{2}$.

Initially, let us suppose that the implied constants in (8) and (17) are of the same order of magnitude, i.e., $k_{0} \approx 1$. Using the contour parameters as defined by (12) and (14), a numerical solution of (18) with $\epsilon \approx 2.2 \times 10^{-16}$ yields that roundoff error becomes significant when $N \approx 24$ (25 in the case of the rational contour (16)). An examination of the convergence curves shown in Section 4 will confirm that $N=24$ is often close to the critical value where roundoff errors start to dominate. More generally, in a multiprecision environment with precision equal to $d$ decimal digits, it follows from (18) that the critical value of $N$ is approximately $1.5 d$.

Considering the general case where $k_{0}$ is not $O(1)$, this critical value of $N$ may be larger. We call this value $N_{*}$, which can be estimated by analyzing the convergence behavior. For $N \leqslant N_{*}$ roundoff error can be neglected, i.e., the main contribution to the error is due to the midpoint approximation. Hence the error behaves like $f(t)-f_{N}(t)=k_{1} e^{-c N}$, where $f_{N}(t)$ denotes the approximation (4). By computing $f_{N}(t)$ for a range of values of $N$, it is straightforward to detect the value of $N$ at which the convergence rate turns from exponential decay to exponential growth. Having determined this critical value $N=N_{*}$ we can insert it into (18). By using the values of the contour parameters defined by (12) and (14), it is possible to compute an approximate value of $k_{0}$.

We use this value of $k_{0}$ to compute new contour parameters for any value of $N$, with $N>N_{*}$. Note that $\zeta(0)=-\sigma+\mu / \alpha$, which depends on $c$ and $\alpha$ via (12). This means the equation in (18) has two unknowns, so we fix $\alpha$ at the value (14). Hence $c$ can be solved by applying a univaritate rootfinding routine to the equation on the right in (18), for a given value of $N\left(>N_{*}\right)$. With $c$ and $\alpha$ known, the new contour parameters for this particular value of $N$ are then computed from (12).

We can justify this approach by looking at the asymptotic behavior of the parameters. Letting $N \rightarrow \infty$ in (18) yields

$$
c=-\log \left(\epsilon / k_{0}\right) N^{-1}+O\left(N^{-3}\right), \quad \zeta(0)=O\left(N^{-3}\right) .
$$

Substitution of these two expressions into the error estimates (8) and (17), respectively, shows that both reduce to $\mathcal{O}(\epsilon)$. Hence errors can be expected to remain at the roundoff level for large $N$.

Because $\zeta(0)=\mathcal{O}\left(N^{-3}\right)$, the contour is not only prevented from moving too far into the right half-plane, it actually moves back to the left (and becomes narrower) when $N>N_{*}$. This means that this strategy is only effective for transforms $F(z)$ whose singularities are exclusively located on the negative real axis. In the next section, we investigate how well it works in practice. 
We remark that a similar roundoff control strategy for the parabola was considered in [Weiio]. A related but different strategy for the hyperbola was proposed in [LFPSo6]. A new strategy that automatically compute a contour of optimal stability for (1) was developed by one of the authors of the present paper. Details can be found in [Din12].

\section{Applications and Comparisons}

In this section we verify the theoretical results of the previous sections by applying the method to various model problems. These include a matrix transform that can be used for the time integration of some parabolic PDEs, as well as two scalar transforms. These two transforms were both taken from applications and both were previously considered as test examples in [Duf93]. This reference also gives series or integral expressions for the inverses, which we used to compute errors.

In all figures, the relative error is plotted against the number of nodes, $N$, in the midpoint approximation (4). For reference, we also plot the theoretically predicted convergence rate $\mathcal{O}\left(e^{-1.358 N}\right)$, as a dash-dot curve. We also remind the reader about the $N$ vs. $2 N$ convention mentioned below (4). When compared to the error curves in [Duf93, Wei1o, WTo7], for example, the values of $N$ on the horizontal axes of the graphs below should therefore be halved.

We start with the model problem (7) and its matrix analogue

$$
F_{1}(z ; \lambda)=(z+\lambda)^{-1}, \quad F_{1}(z ; A)=(z I+A)^{-1} .
$$

Here $\lambda>0$ and $A$ is a symmetric positive definite matrix, with $I$ the identity matrix of the same size.

The matrix version arises, for example, when semi-discretizations of the heat equation are solved by Laplace transform techniques [GMo5, LFPo4, SSTo3]. Consider

$$
\mathbf{u}_{t}+A \mathbf{u}=\mathbf{0} \quad \Longrightarrow \quad z \mathbf{U}(z)-\mathbf{u}_{0}+A \mathbf{U}(z)=\mathbf{0} \quad \Longrightarrow \quad \mathbf{U}(z)=F_{1}(z ; A) \mathbf{u}_{0},
$$

where $A$ is a $J \times J$ matrix representation of the negative Laplacian, $\mathbf{u}(\mathrm{t})$ an $J \times 1$ vector of unknowns with $\mathbf{U}(z)$ its Laplace transform, and $\mathbf{u}_{0}$ the initial condition. The inverse formula ( 1 ) yields

$$
\mathbf{u}(t)=\exp (-A t) \mathbf{u}_{0}=\frac{1}{2 \pi i} \int_{C} e^{z t}\left(F_{1}(z ; A) \mathbf{u}_{0}\right) d z .
$$

In this case the singularities of the transform are the negatives of the eigenvalues $\lambda$ of $A$, which are located on the negative real axis because of the assumptions on $A$. The right-hand side is approximated by the midpoint sum (4), where each quadrature node requires the solution of a linear system with coefficient matrix $\left(z\left(\theta_{k}\right) I+A\right)$ and right-hand side $\mathbf{u}_{0}$.

Our test example is the heat equation $u_{t}-0.01 \nabla^{2} u=0$ on $[0,1] \times[0,1]$, supplemented with homogeneous Dirichlet boundary conditions. We take $A$ as the familiar block-tridiagonal matrix based on the 5-point finite difference approximation to the negative of the Laplacian, which is known to be positive definite. The right-hand side $\mathbf{u}_{0}$ is taken to be random, and the reference solution $\mathbf{u}(t)$ was computed using the spectral decomposition of $A$, which is explicitly known. 
The results for this example are shown in Figure 4. Note that we have plotted the error here in the computed value of $\exp (-A t) \mathbf{u}_{0}$, i.e., only the temporal error. The error in the actual solution of the PDE is not shown but it would not reach such small values unless a super accurate space discretization is used.
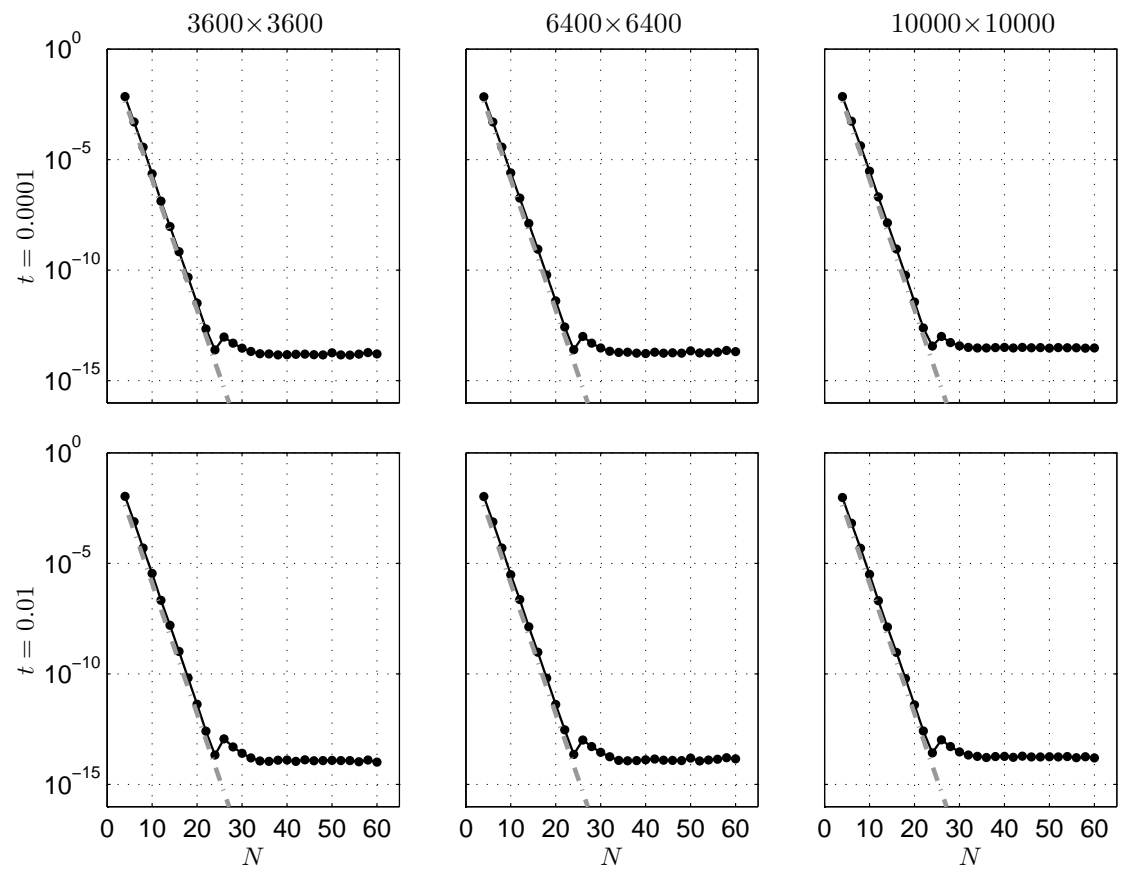

FIGURE 4. Relative errors in the $\infty$-norm when $\exp (-A t) \mathbf{u}_{0}$ is approximated by applying the midpoint sum (4) to the contour integral (20). For smaller values of $N$ the contour (15) was used, while for larger $N$ the roundoff control procedure of Section 3 was used. The three columns correspond to three sizes of $A$, while the two rows correspond to two values of $t$. The dashed line represents the theoretical predicted convergence rate $\mathcal{O}\left(e^{-1.358 N}\right)$.

Figure 4 confirms that the modified Talbot contour converges as predicted by the error analysis of Section 2, namely $\mathcal{O}\left(e^{-1.358 N}\right)$ in all cases shown. Ten-digit accuracy is reached with $N=18$ or fewer nodes, i.e., no more than 9 transform evaluations are required (each of which involves the solution of a sparse linear system of the indicated dimension). Almost full accuracy is reached for all $N>24$.

The roundoff control strategy described in the previous section is also seen to work well in practice. To implement it, we set in the computations of Figure 4 the value $k_{0}=1$ and used the alternate parameters for all $N \geqslant 24$.

The remaining transforms in this section are scalar problems from actual applications, as collected in [Duf93]. As an example of a transform with a series of poles on the negative real axis we consider

$$
F_{2}(z)=\frac{(100 z-1) \sinh \left(\frac{1}{2} \sqrt{z}\right)}{z(z \sinh (\sqrt{z})+\sqrt{z} \cosh (\sqrt{z}))} .
$$


This is Test 2 in [Duf93], where it was noted that the apparent square root singularity can be removed by expanding the hyperbolic functions. We also consider Test 3 in [Duf93], namely

$$
F_{3}(z)=\frac{1}{z} \exp \left(-r \sqrt{\frac{z(1+z)}{1+c z}}\right), \quad r>0, c>0 .
$$

This transform has a pole at $z=0$, an essential singularity $z=-1 / c$ as well as branch point singularities at $z=0$ and $z=-1$. With appropriate definition of the branch cuts, this transform is analytic everywhere off the negative real axis.

Numerical results for transforms $F_{2}(z)$ and $F_{3}(z)$ are presented in Figures 5 and 6 , respectively. (The values of $t$ in these figures were chosen to match those in [Duf93].) Again we see empirical confirmation of the theoretical $\mathcal{O}\left(e^{-1.358 \mathrm{~N}}\right)$ convergence rate and the effectiveness of the roundoff control strategy.
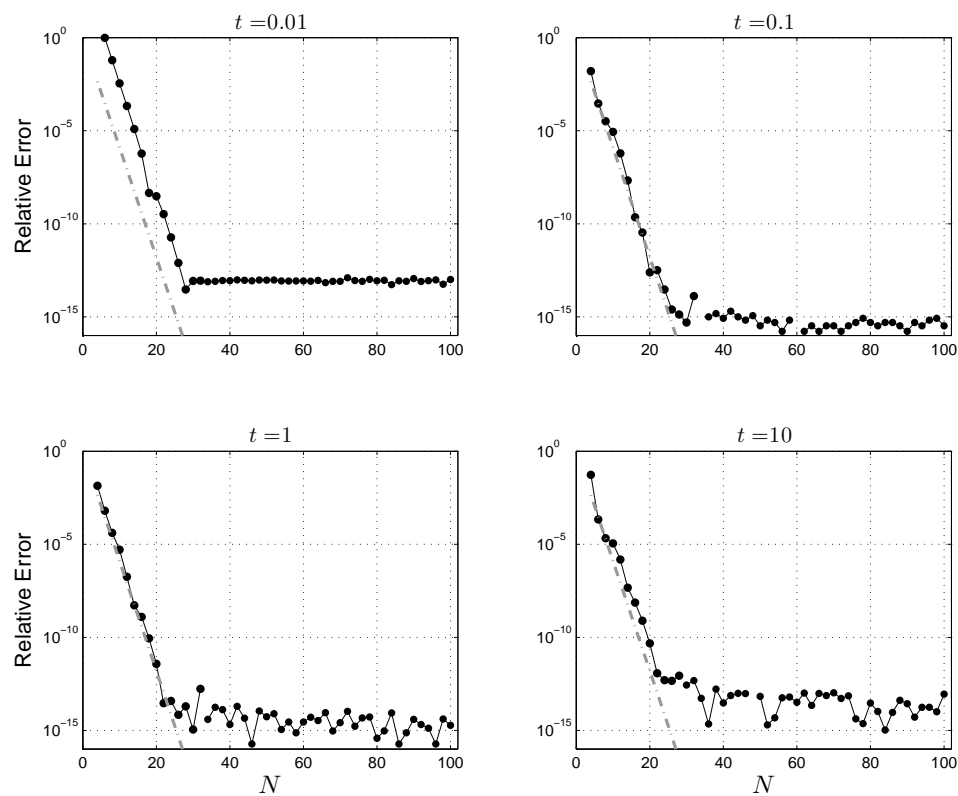

Figure 5. Relative error in the inversion of transform $F_{2}(z)$. For smaller values of $N$ the contour (15) was used, while for larger $N$ the roundoff control procedure of Section 3 was implemented.

A comparison with the results presented in [Duf93] shows a faster rate of convergence here. For example, to achieve ten-digit accuracy at $t=1$, the midpoint sum (4) requires $N=18$, i.e., 9 transform evaluations, for both transforms $F_{2}$ and $F_{3}$. In contrast, the algorithm used in [Duf93] achieves ten-digit accuracy using 17 and 13 transform evaluations for $F_{2}$ and $F_{3}$, respectively. The corresponding number of transform evaluations for the method of [AVo4] is 15 and 14 .

Note that $F_{3}(z)$ is an example for which the error analysis of Section 2 can be less reliable. The reason is its rapid decay as $z \rightarrow+\infty$, particularly if $r \gg 1$; recall 

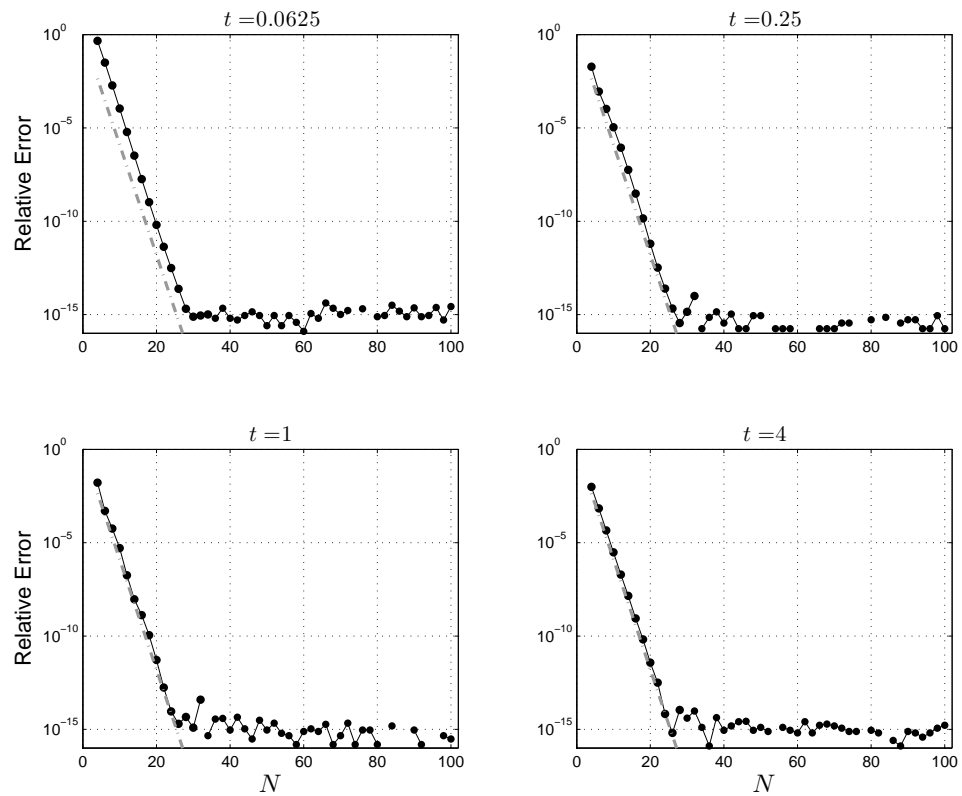

Figure 6. Same as Figure 5 but the transform is $F_{3}(z)$, with $c=0.4$ and $r=0.5$.

the discussion below (8). To see what happens, we show in Figure 7 convergence curves in the case $r=3$. Asymptotically, the convergence rate is still $\mathcal{O}\left(e^{-1.358 N}\right)$ (as indicated by the dash-dot curve) but the implied constant has increased by some orders of magnitude. This also means that the critical value $N$ where roundoff error becomes significant has now increased from $N=24$ to about $N=38$ and $N=32$, respectively, in the cases $t=1$ and $t=4$. Nevertheless, the roundoff control strategy of Section 3 is reasonably successful in preventing any catastrophic error growth.
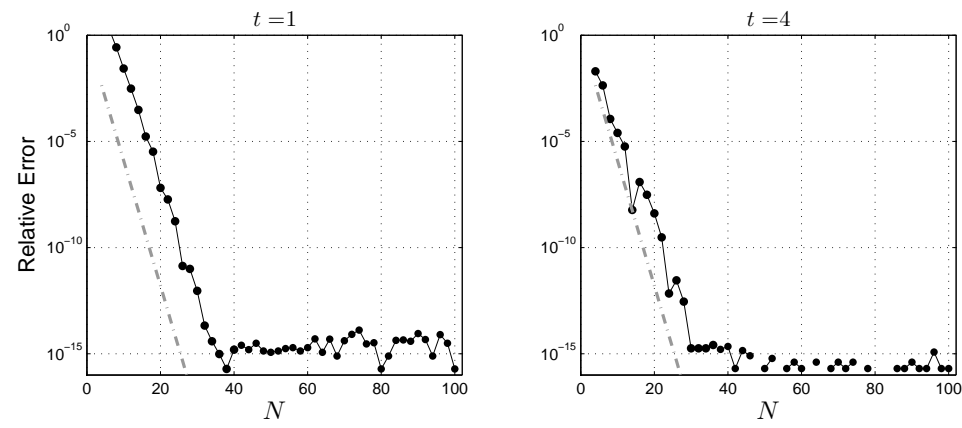

FIGURE 7. Same as Figure 5 but the transform is $F_{3}(z)$, with $c=0.4$ and $r=3$. 


\section{Conclusions}

A truncated version of Talbot's famous contour for numerical Laplace transform inversion was analyzed. For the case where the singularities of the transform are located on the negative real axis, a new type of error analysis was proposed and used to derive optimal contour parameters. With these parameters an exponentially convergent method is obtained, with decay constant better than other known contours. In addition, a roundoff control strategy was proposed for improved numerical stability when the number of nodes in the quadrature scheme gets large. Numerical experiments on transforms taken from actual applications confirmed the accuracy of the optimal error estimates as well as the efficiency of the roundoff control scheme.

We believe the improvements to Talbot's method suggested in this paper should be considered for inclusion in future versions of software such as those mentioned in Section 1. Our own MATLAB code that was used in generating the numerical results of Section 4 can be downloaded from [DW14].

Acknowledgment. The research of BD was supported by the DFG Collaborative Research Center TRR 109, "Discretization in Geometry and Dynamics." He further acknowledges support from the graduate program TopMath of the Elite Network of Bavaria and the TopMath Graduate Center of TUM Graduate School at Technische Universität München. The research of JACW was supported the National Research Foundation of South Africa.

Folkmar Bornemann and Nick Trefethen both offered valuable suggestions, as have two anonymous referees.

\section{REFERENCES}

[AVo2] AвATE, J. ; VALKó, P. P.: NumericalLaplaceInversion. In: ht tp: // library. wolfram. com/ infocenter/MathSource/4738/ (2002) 2

[AVo4] AватE, J. ; VAlKó, P. P.: Multi-precision Laplace transform inversion. In: International Journal for Numerical Methods in Engineering 6o (2004), Nr. 5, S. 979-993 2, 3, 13

[But57] Butcher, J. C.: On the numerical inversion of Laplace and Mellin transforms. Salisbury, Australia, 1957 (Conference on Data Processing and Automatic Computing Machines) 1, 2

[Din12] Dingfelder, B.: Raten- und stabilitätsoptimierte Algorithmen zur Berechnung eines Integrals mit Hankelintegrationsweg. Germany, Technische Universität München, Bachelor's thesis, September 201211

[Duf93] DuFF, D. G.: On the numerical inversion of Laplace transforms: comparison of three new methods on characteristic problems from applications. In: ACM Trans. Math. Software 19 (1993), September, Nr. 3, S. 333-359. - ISSN oog8-3500 2, 11, 12, 13

[DW14] Dingfelder, B. ; Weideman, J. A. C.: ModifiedTalbot. In: http: //arxiv org/e-print/ $1304.2505 v 3$ (2014) 15

[GMo5] GAvriLyuK, I. P. ; MAKarov, V. L.: Exponentially convergent algorithms for the operator exponential with applications to inhomogeneous problems in Banach spaces. In: SIAM J. Numer. Anal. 43 (2005), Nr. 5, S. 2144-2171. - ISSN o036-1429 2, 11

[Kre98] Kress, R.: Numerical analysis. New York : Springer-Verlag, 1998. - xii+326 S. - ISBN o-387$98408-95,6$

[LFPo4] López-Fernández, M. ; Palencia, C.: On the numerical inversion of the Laplace transform of certain holomorphic mappings. In: Appl. Numer. Math. 51 (2004), Nr. 2-3, S. 289-303. ISSN $0168-92742,11$

[LFPSo6] López-Fernández, M. ; Palencia, C. ; Schädle, A.: A spectral order method for inverting sectorial Laplace transforms. In: SIAM J. Numer. Anal. 44 (2006), Nr. 3, S. 1332-1350. - ISSN o036-1429 11 
[McC13] McClure, T.: talbot_inversion. In: http://www. mathworks. com/matlabcentral/ fileexchange/39035-numerical-inverse-laplace-transform/ (2013) 2

[MR9o] Murli, A. ; Rizzardi, M.: Algorithm 682: Talbot's method for the Laplace inversion problem. In: ACM Trans. Math. Software 16 (1990), Juni, Nr. 2, S. 158-168. - ISSN oog8-3500 2

[Riz95] RizzARDI, M.: A modification of Talbot's method for the simultaneous approximation of several values of the inverse Laplace transform. In: ACM Trans. Math. Software 21 (1995), Nr. 4, S. 347-371. - ISSN oog8-3500 5

[SSTo3] SheEn, D. ; SLOAN, I. H. ; ThOmÉE, V.: A parallel method for time discretization of parabolic equations based on Laplace transformation and quadrature. In: IMA J. Numer. Anal. 23 (2003), Nr. 2, S. 269-299. - ISSN 0272-4979 2, 11

[Stro7] Strang, G.: Computational science and engineering. Wellesley, MA : Wellesley-Cambridge Press, 2007. - xii+716 S. - ISBN 978-0-9614088-1-7; 0-9614088-1-2 3

[Tal79] Тацвот, A.: The accurate numerical inversion of Laplace transforms. In: J. Inst. Math. Appl. 23 (1979), Nr. 1, S. 97-120. - ISSN 0020-2932 1, 2, 3, 9

[Treo6] Private communication 3

[TWSo6] Trefethen, L. N. ; Weideman, J. A. C. ; Schmelzer, T.: Talbot quadratures and rational approximations. In: BIT 46 (2006), Nr. 3, S. 653-670. - ISSN ooo6-3835 1, 3, 9

[Weio6] Weideman, J. A. C.: Optimizing Talbot's contours for the inversion of the Laplace transform. In: SIAM J. Numer. Anal. 44 (2006), Nr. 6, S. 2342-2362. - ISSN oo36-1429 2, 3, 5, 6, 8, 9

[Weiıo] WeIDEMAN, J. A. C.: Improved contour integral methods for parabolic PDEs. In: IMA J. Numer. Anal. 30 (2010), Nr. 1, S. 334-350. - ISSN 0272-4979 10, 11

[WTo7] Weideman, J. A. C. ; Trefethen, L. N.: Parabolic and hyperbolic contours for computing the Bromwich integral. In: Math. Comp. 76 (2007), Nr. 259, S. 1341-1356. - ISSN o025-5718 2, 3, 5, 6,11

Zentrum Mathematik - M3, Technische Universität München, 8029o München, Germany

E-mail address: dingfelder@mytum.de

Department of Mathematical Sciences, University of Stellenbosch, Stellenbosch 76oo, South Africa

E-mail address: weideman@sun.ac.za 